\section{PROCESOS DE DESARROLLO URBANO-REGIONAL Y EXCLUSIÓN TERRITORIAL: Nuevas formas de urbanización en el área metropolitana de Valparaíso. Estudio de caso ciudad de Curauma $^{1}$}

Pablo Mansilla Q. ${ }^{2}$

Manuel Fuenzalida D. ${ }^{3}$

\section{Resumen}

El retroceso del Estado en la planificación del desarrollo urbano y el rol asumido por parte del sector privado en la producción del espacio urbano ha abierto la posibilidad a la generación de nuevas formas de urbanización, que modifican las estructuras territoriales tradicionales. Los cambios que se han generado a partir de estas dinámicas resultan evidentes en el caso de las grandes ciudades Chilenas, constituyéndose como huellas claras de los discursos y estrategias políticas.

El presente artículo aborda el contexto en el que se han desarrollado los procesos de urbanización en
URBAN-REGIONAL DEVELOPMENT PROCESSES AND TERRITORIAL EXCLUSION: New forms of urbanization in the metropolitan area of Valparaíso. Case study of the city of Curauma ${ }^{1}$

Pablo Mansilla Q. ${ }^{2}$

Manuel Fuenzalida D. ${ }^{3}$

\section{Abstract}

The decline of the role of the State in urban development planning and the participation of the private sector in the production of urban space offers the possibility of new forms of urbanization, modifying traditional territorial structures. Changes generated by these dynamics are evident in the case of major Chilean cities, which show obvious traces of political discourses and strategies.

By taking the city of Nueva Ciudad de Curauma as reference, the present article addresses the 
el área metropolitana de Valparaíso tomando como referencia la Nueva Ciudad de Curauma. Para ello contextualiza los escenarios que hacen posible la creación de Curauma, el estado actual en el que se encuentra, se define la tipología de urbanización cerrada que representa y el rol actual que cumple en el contexto del Área Metropolitana de Valparaíso. Finalmente se extienden algunas reflexiones acerca de los procesos de exclusión territorial que se reproducen a partir de estas nuevas formas de urbanización.

\section{PALABRAS CLAVE: PLANIFICACIÓN URBANA, CIUDADES CERRADAS, CENTRALIDADES, EXCLUSIÓN TERRITORIAL.}

Fecha de recepción: 13.11.2009

Fecha de aceptación: 30.07.2010

1 Este artículo presenta los primeros resultados obtenidos du rante el año 2009 por el Laboratorio de Investigación Ciudad y Territorio del Instituto de Geografía de la Pontificia Universidad Católica de Valparaíso, el cual forma parte de una serie de investigaciones mediante las cuales se pretenden identificar las principales transformaciones urbanas que ha experimentado durante las últimas décadas el área Metropolitana de Valparaíso (Chile) y los impactos socio espaciales que se han desprendido a partir de estas dinámicas.

2 Chileno. Geógrafo, Magíster (c) en Geografía Universidad de Chile. Investigador Laboratorio Ciudad y Territorio, Instituto de Geografía, Pontificia Universidad Católica de Valparaíso. Chile. Correo electrónico: pablo.manq@gmail.com

3 Chileno. Geógrafo, Doctor Universidad Autónoma de Madrid Profesor Asociado Instituto de Geografía, Pontificia Universidad Católica de Valparaíso. Chile.

Correo electrónico: manuel.fuenzalida@ucv.cl context in which the processes of urbanization in the Metropolitan Area of Valparaíso take place. To that effect, the circumstances that made possible the creation of Curauma; its current state; the typology of gated residential community that the city represents; and its current role in the Metropolitan Area of Valparaíso are contextualized. The article ends with reflections on territorial exclusion processes triggered by these new forms of urbanization.

\section{KEYWORDS: URBAN PLANNING, GATED RESIDENTIAL COMMUNITY, CENTRALITIES, TERRITORIAL EXCLUSION.}

Received: 13.11.2009

Accepted: 30.07 .2010

1 This article shows the first results achieved by the Laboratorio de Investigación Ciudad y Territorio, Institute of Geography, Pontificia Universidad Católica de Valparaíso. It is part of a series of research intended to identify the main urban transformations that the Metropolitan Area of Valparaíso (Chile) has experienced over the last decades and the socio-spatial impacts caused by these dynamics.

2 Chilean. Geographer, Master in Geography, Universidad de Chile. Researcher, Laboratorio de Investigación Ciudad y Territorio, Institute of Geography, Pontificia Universidad Católica de Valparaíso. Chile.

Email: pablo.manq@gmail.com

3 Chilean, Geographer, Doctor, Universidad Autónoma de Madrid. Associated profesor, Institute of Geography, Pontificia Universidad Católica de Valparaíso. Chile.

Email: manuel.fuenzalida@ucv.cl 


\section{Introducción}

Las evidencias empíricas obtenidas a partir de diversos estudios urbanos ${ }^{4}$ coinciden en que las transformaciones que han experimentado durante la última década las grandes ciudades de América Latina, encuentran su principal explicación en la profundización de los procesos asociados a la globalización y a las estrategias neoliberales que en ellas se han implementado en la producción del espacio urbano. En este contexto, los Estados han asumido un menor peso normativo en la planificación urbana, dejando abierta la posibilidad para que el desarrollo urbano se rija por el libre juego de la oferta y la demanda del uso de suelo, lo cual ha tenido como consecuencia principal un aumento de la segregación social y fragmentación de las ciudades.

En Chile, la mayor parte de los estudios en esta materia se han llevado a cabo en el Área Metropolitana de Santiago, debido a que en ella el alto dinamismo del mercado inmobiliario ha generado que estos procesos se materialicen con más fuerza y que se hayan caracterizado por el desarrollo de grandes emprendimientos, de tipo extensivo y discontinuo, que dan lugar a diversas tipologías de ciudades cerradas. Sin embargo, es importante resaltar que este modelo también se ha difundido hacia otras grandes urbes metropolitanas como las de Concepción y Valparaíso.

En esta última región, la urbanización posee un desarrollo extremadamente dinámico y de ritmo acelerado, donde el Área Metropolitana de Santiago (AMS) y el Área Metropolitana de Valparaíso (AMV) se transforman en factores sinergéticos claves en el proceso de urbanización regional, debido a que la escasa superficie urbanizable y el aumento de los valores de suelo, sumado al aumento de la primacía urbana, han contribuido al deterioro de la calidad de vida de sus habitantes en múltiples aspectos. De esta forma la demanda de espacios naturales y lugares que presenten mejores condiciones para el desarrollo personal y de la familia, hacen que los grupos socio económicos medios y altos decidan desplazarse fuera de la ciudad. Esta dinámica ha potenciado la modificación de los usos de suelo tradicionales que se habían mantenido en algunas zonas o localidades rurales, generando presiones tanto sobre sus características naturales, como sobre las realidades locales en cuanto a los aspectos sociales y culturales. Precisamente es en las áreas de alta valorización paisajística donde se

4 Warner y Negrete, 2001-2002; Janoschka, 2002; Hidalgo y Gonzáles, 2004; Hidalgo y Borsdorf, 2005; Hidalgo, Borsdorf y Sánchez, 2005; Parnreiter, 2005; Hidalgo et al, 2007. 
encuentran enfocados los intereses de desarrollo inmobiliario, ya que éstos representan una excelente estrategia de marketing, transformando las condiciones naturales de estos lugares en bienes transables en el mercado.

En este contexto, la influencia que ha tenido la innovación en infraestructura de transporte sobre las dinámicas urbanas de las ciudades intermedias y de los nuevos asentamientos que se han desarrollado en las últimas décadas en la Región de Valparaíso, resulta ser un proceso evidente y que de cierta forma permite el desarrollo de proyectos inmobiliarios como el que nos interesa analizar aquí: $\mathrm{Cu}$ rauma. Sobre esta idea, Borsdorf ${ }^{5}$ menciona que el proceso de difusión de la ciudad ha encontrado un correlato con la construcción de autopistas intraurbanas modernizadas y ampliadas por medio de capitales privados, las cuales han mejorado la accesibilidad y la conectividad de las zonas periféricas y periurbanas, volviéndolas a ser atractivas para las clases medias y altas

Uno de los proyectos más representativos de las nuevas formas de urbanización que se han desprendido de la adopción del enfoque neoliberal en el Área Metropolitana de Valparaíso (AMV)

5 BORSDORF, Axel, 2003.

106 revista invi № 69 / Agosto 2010 / Volumen № 25: 103-123 es la nueva ciudad de Curauma, emplazada en la comuna de Valparaíso, entre el AMV y el AMS, a un costado del sector de Placilla ${ }^{6}$ y de la Reserva Nacional Lago Peñuelas. El proyecto ha sido planificado y desarrollado totalmente por el sector privado y ha recibido el apoyo tanto de los gobiernos locales como por parte de instituciones del Estado, los cuales han contribuido mediante la inversión en infraestructura vial y de edificios institucionales ${ }^{7}$ para aportar a la consolidación de esta nueva ciudad. Asimismo, el municipio ha facilitado el accionar de las inmobiliarias habilitando mediante común acuerdo un plan seccional que integra a Curauma en el plan Regulador de la Comuna de Valparaíso. De esta forma, el proyecto contempla una zona urbana, con más de dieciocho barrios, la consolidación de una zona industrial en la zona aledaña a la Reserva Nacional Lago Peñuelas, la localización de algunos servicios privados que permiten abastecer en parte las demandas de sus habitantes y un mega proyecto impulsado por la Pontificia Universidad Católica de Valparaíso, la cual ha realizado millonarias inversiones para la construcción de un campus que re-localice y reúna a gran parte de las facultades universitarias y oficinas administrativas que la componen. Asimismo,

6 Sector popular perteneciente a la comuna de Valparaíso que ha demandado históricamente mejoras en cuanto a la calidad de sus servicios y equipamientos que les permitan disponer de las condiciones básicas para desarrollar una buena calidad de vida.

$7 \quad$ A modo de ejemplo, el Edificio Tecnológico de la Corporación de Fomento de la Producción (CORFO). 
en la actualidad se encuentra en fase de construcción el condominio Puerto Esperanza que contempla más de 880 viviendas sociales, temática que ha causado gran controversia entre los habitantes de Curauma, con opiniones divergentes que aceptan y rechazan la llegada de estos nuevos vecinos.

El fuerte contraste que genera Curauma con las características tradicionales que había mantenido este sector del AMV, así como el desarrollo de una serie de conflictos de intereses que se han generado a partir del fuerte incremento de población y de las demandas por servicios, nos hacen reflexionar acerca de la sustentabilidad de los modelos de desarrollo urbano que se están desplegando en la actualidad, las formas en que las instituciones administrativas y privadas operan en la construcción del espacio y finalmente, quienes son los beneficiados y excluidos de estos procesos.

\section{La nueva ciudad de Curauma en el contexto del desarrollo urbano en el área metropolitana de Valparaíso}

La urbanización en el AMV, desde sus inicios, se ha caracterizado por un desarrollo espontáneo, llegando a calificar en algunos casos según Warner y
Negrete $^{8}$, como situaciones dramáticas de urbanización a partir de la falta de control ya sea de manera formal o informal. Ambos autores identifican que el factor explicativo de mayor peso en la falta de control del desarrollo urbano, guarda relación con la fuerte adopción del enfoque neoliberal durante la dictadura militar y en la gradualidad con que los gobiernos democráticos han restituido y generado nuevas normas de regulación territorial, dejando espacios de tiempo abiertos para que el sector inmobiliario pueda concretar proyectos masivos que han impactado directamente sobre el patrimonio natural y cultural de las ciudades del AMV.

En el periodo anterior a la dictadura, el Estado se caracterizó por asumir un rol director en el proceso de urbanización de las ciudades Chilenas, situación que se modifica durante la dictadura militar con la adopción del enfoque neoliberal, generando un cambio en el modelo de desarrollo y un retroceso de la función del Estado en materia de regulación urbana. Hidalgo et. al. ${ }^{9}$, profundizan esta idea mencionando que durante este periodo el Estado asume un menor peso normativo y las políticas se tienden a encontrar basadas en el libre juego de la oferta y la demanda, haciendo que el suelo pase a ser una mercancía más que se transa en un mercado abierto. De esta forma, los agentes inmobiliarios asumen el rol de transformarse en

8 WARNER, Kee y NEGRETE, Jorge. 2001-2002.

9 HIDALGO et. al. (2007). 
actores principales del proceso de desarrollo urbano. La materialización más clara de esta delegación de poder ocurre en el año 1979, donde se decreta la liberalización del mercado de suelo y se eliminan los límites urbanos establecidos en los instrumentos de regulación territorial. De esta forma, el Estado pasa a asumir un rol adaptativo en el proceso de desarrollo urbano, posicionándose como espectador y sólo interviniendo para dar solución a los conflictos y demandas que la actividad inmobiliaria va generando a su paso.

Estas modificaciones en el rol del Estado en la planificación del desarrollo urbano impactan directamente sobre el desarrollo de las grandes ciudades Chilenas, tal como lo mencionan Hidalgo y Gonzáles $^{10}$, las políticas y reformas económicas que se producen durante la dictadura, que postulan que el suelo es un bien cuyo funcionamiento debía regirse por la ley de la oferta y demanda, generan que las urbanizaciones en la región metropolitana adquieran un carácter extensivo y discontinuo.

Posteriormente, con el retorno de los gobiernos democráticos, aunque se intenta regular nuevamente el desarrollo urbano, esta situación no se revierte, en este sentido Warner y Negrete ${ }^{11}$ señalan que si bien en la actualidad se ha logrado concretar una regulación frente al desarrollo urbano, ésta aparece como mucho más liberal y descontrolada que

10 HIDALGO Y GONZÁLES, 2004 (Citando a Hidalgo y Arenas, 2003). 11 WARNER, Kee y NEGRETE, Jorge, 2001-2002.

108 revista invi № 69 / Agosto 2010 / Volumen № 25: 103-123 las regulaciones anteriores a la dictadura. De esta forma, definen al proceso de regulación como "un procedimiento formal más que sustantivo", debido a que cada proyecto debe pasar por una serie de burocracias que no exigen demasiado, por consiguiente, el Estado y sus instituciones con competencias en la planificación y desarrollo urbano, han pasado a ser espectadores del proceso de urbanización de las ciudades Chilenas, dejando abierto el campo de acción al sector privado.

En este contexto nace Curauma, un proyecto de ciudad planteado desde la década de 1970, pero que recién comenzó a materializarse a partir del año 1996, sentando uno de los precedentes a nivel nacional en cuanto a mega proyectos urbanos privados. Los terrenos en que se encuentra emplazado este proyecto antiguamente poseían un uso forestal y conformaban parte del fundo "Forestal Valparaíso", propiedad de la misma empresa.

El proyecto se encuentra localizado en la Región de Valparaíso, en la comuna del mismo nombre. Su cercanía a los dos grandes centros urbanos articuladores de la Macro Zona Central, a $15 \mathrm{Km}$. del AMV y a unos $75 \mathrm{Km}$ del AMS sumado a su emplazamiento a un costado de la ruta 68 , le permiten tener una alta conectividad con los principales centros que estructuran la organización urbano regional. La cercanía con estos dos grandes cen- 
tros poblados es la principal fortaleza del proyecto inmobiliario, ya que a las personas que decidan acceder a una vivienda en Curauma, les posibilita adoptar un estilo de vida de "commuter"12.

Asimismo, Curauma se localiza en el área colindante a la localidad de Placilla, un sector popular de la comuna de Valparaíso que alberga aproximadamente 10.811 Hab., según los antecedentes del Censo 2002, que le permiten satisfacer en parte las necesidades que posee la población de Curauma.

El proyecto se extiende sobre 4.300 Has., que antiguamente correspondían a los fundos "Las Cenizas" y "La Luz", los cuales poseen una extensión total de 2.012 Has. y 2.313 Has. Respectivamente (ver tabla 1). La extensión de superficie que posee el proyecto es sustantiva si se observa que ésta abarca una superficie mayor que la ciudad de Viña del Mar (3.060 Has.). Asimismo, Curauma representa un 34,4\% del suelo disponible para extensión urbana del AMV. Del mismo modo, llama la atención la extensión de las áreas destinadas especialmente a uso natural en el proyecto, las cuales se perfilan como otra de las estrategias de marketing de las inmobiliarias para captar compradores. En el caso del Fundo La Luz estas áreas contemplan un 23\%

12 Concepto que hace referencia a aquel segmento de la población que se moviliza hacia centros de trabajo que se localizan en otras ciudades, como Santiago, Valparaíso o Viña del Mar, los cuales realizan movimientos pendulares que contemplan su desplazamiento durante el día hasta estos centros durante la jornada laboral y su regreso durante la noche al lugar de residencia. del total de su superficie y en el caso del Fundo Las Cenizas un 29,8\%.

\section{TOTAL DE HECTÁREAS DISPONIBLES PARA EL PROYECTO CURAUMA SEGÚN USO DESTINADO}

\begin{tabular}{lrrrrr} 
Fundo & $\begin{array}{l}\text { TOTAL } \\
\text { (Has.) }\end{array}$ & $\begin{array}{l}\text { Área disponible para } \\
\text { uso residencial, } \\
\text { industrial y comercial } \\
\text { (Has.) }\end{array}$ & $\begin{array}{l}\text { Áreas Verdes, } \\
\text { Lago, Áreas }\end{array}$ \\
& & $\mathrm{n}^{0}$ & $\begin{array}{l}\text { Protegidas } \\
\text { (Has.) }\end{array}$ \\
Total & 4.325 & 3.193 & 73,8 & 1.132 & 26,2 \\
\hline La Luz & 2.313 & 1.781 & 77,0 & 532 & 23,0 \\
\hline Las Cenizas & 2.012 & 1.412 & 70,2 & 600 & 29,8 \\
\hline
\end{tabular}

TABLA 1

Fuente: Curauma S.A. Individual Balance Sheet as of September 30 th, 2006

Actualmente el proyecto se ha desarrollado sólo en el sector del fundo La Luz, mientras que en el sector de Las Cenizas recién se está comenzando a proyectar la construcción de nuevas viviendas. Este último proyecto espera tener un éxito superior debido a que, por un lado, se encuentra más cercano a las ciudades de Valparaíso y Viña del Mar y por otro, el fundo Las Cenizas se encuentra dentro 
del área decretada como zona de Urbanización de Alta Densidad por el plan regulador, el que acepta la construcción de 70 viviendas por hectárea, lo que potencialmente permitiría la construcción de 98.000 viviendas $^{13}$.

Según las estimaciones realizadas por la Sociedad Curauma, en septiembre del 2006 residían en la ciudad aproximadamente unos 13.000 habitantes, proyectando un incremento de población en 4.000 nuevos residentes por año. Asimismo, Hidalgo y Borsdorf ${ }^{14}$ mencionan que en el horizonte de 25 años, cuando se prevé que estará construido totalmente el proyecto, espera acoger a una población entre 150.000 y 200.000 habitantes.

La importancia que posee Curauma dentro de la oferta inmobiliaria del AMV, resulta evidente al observar que este proyecto se perfila como el principal centro inmobiliario en captación de compradores de nuevas residencias, cubriendo aproximadamente el 50\% de la demanda de viviendas. Además, el rápido crecimiento que ha experimentado desde el año 1997 queda evidenciado al observar ${ }^{15}$ que inicialmente parte con una venta anual inferior a las 200 unidades, mientras que para el año 2005 se venden 1.302 viviendas nuevas, situación que se proyecta para el año 2006 en un leve incremento a un total de 1.350 viviendas.

13 Revista Ercilla.

14 HIDALGO, Rodrigo; BORSDORF, Axel, 2005

15 Según los datos proporcionados por Curauma S.A.

110 revista invi № 69 / Agosto 2010 / Volumen № 25: 103-123
La estrategia implementada por el empresario Manuel Cruzat, para llevar a cabo la realización del proyecto inmobiliario, consiste en la gestión del proyecto y la venta de macro lotes a otras inmobiliarias, las cuales a su vez se encargan de llevar a cabo el diseño y la construcción de sus propios proyectos y venderlos. A través de esta estrategia, $\mathrm{Cu}$ rauma S.A. logra conseguir el capital necesario para llevar a cabo el desarrollo de esta nueva ciudad.

En la actualidad existen 18 proyectos residenciales gestionados por diferentes inmobiliarias, los cuales se desarrollan en dos zonas denominadas $\mathrm{Cu}$ rauma Norte y Curauma Sur, separadas por paños de suelo sin uso actual.

En el sector Norte de Curauma se han desarrollado 10 proyectos inmobiliarios, los que poseen valores que varían entre las 630 UF $^{16}$ y las 2700 UF, de éstos, 6 corresponden a condominios cerrados que garantizan seguridad durante las 24 horas, poseen control en la puerta de acceso y algunos otros mecanismos de vigilancia.

Asimismo, uno de los elementos que resalta en el proyecto inmobiliario Curauma, es que contempla entre sus intereses aportar a fortalecer la "heterogeneidad socio económica", para ello potencia el desarrollo de algunos proyectos de vivienda social en

16 En Chile, la Unidad de Fomento (UF) es una unidad de cuenta reajustable de acuerdo con la inflación. Al 30-11-2009 alcanzó los $\$ 21.017,51$ pesos chilenos (CLP), equivalentes a \$42,39 dólares americanos (USD)

ARTÍCULO: Procesos de desarrollo urbano-regional y exclusión territorial: Nuevas formas de urbanización en el área metropolitana de Valparaíso. Estudio de caso ciudad de Curauma /

Pablo Mansilla O., Manuel Fuenzalida D. 


\section{ESTRUCTURA GENERAL DE CURAUMA}

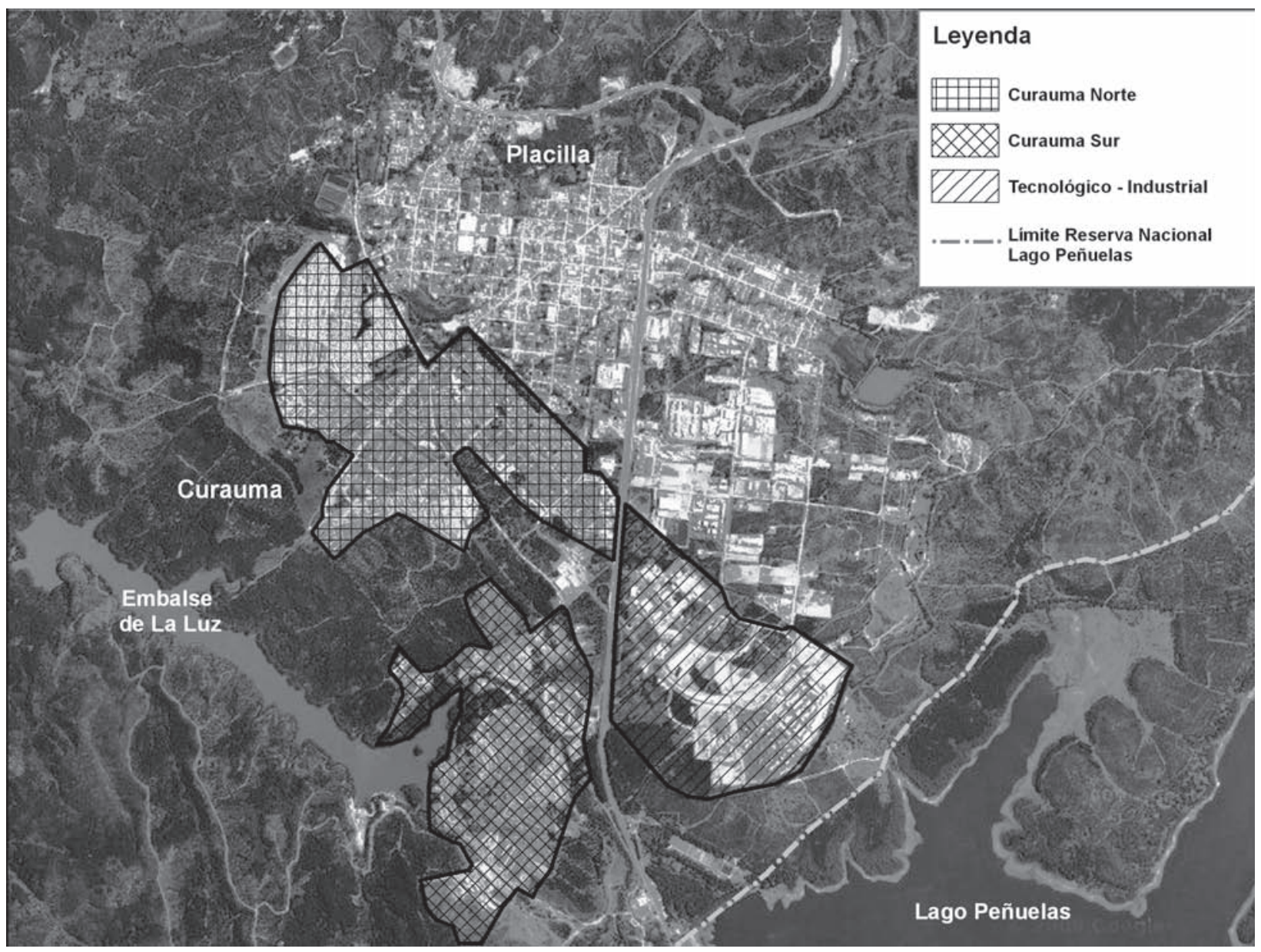

FIGURA 1

Fuente: Elaboración Propia.

ARTíCULO: Procesos de desarrollo urbano-regional y exclusión territorial: Nuevas formas de urbanización en el área metropolitana de Valparaíso. Estudio de caso ciudad de Curauma/

revista invi № 69 / Agosto 2010 / Volumen № 25: 103-123 111

Pablo Mansilla 0., Manuel Fuenzalida D. 
el sector de Curauma Norte, como el proyecto de Cardenal Samore, emplazado en un sector inmediatamente colindante a Placilla. En él es posible encontrar viviendas sociales que van desde las 630 UF a 939 UF y que poseen subsidio habitacional del Estado. Estas viviendas son las que poseen la menor cantidad de metros cuadrados construidos de todos los proyectos que localizan en Curauma y presentan los mayores índices de concentración con más de 1150 viviendas, cifra que supera ampliamente la cantidad de viviendas construidas por los otros proyectos inmobiliarios. Actualmente en la misma zona se encuentra en etapa de desarrollo un segundo proyecto de viviendas sociales denominado "Puerto Esperanza", el cual destinará más de 880 viviendas sociales nuevas, ante las cuales se estima la llegada de aproximadamente 4.000 habitantes nuevos a Curauma. Situación que no ha estado fuera de un intenso debate, en el que algunas organizaciones vecinales de Curauma han manifestado su malestar ante las autoridades, aludiendo a los impactos negativos que este proyecto podría generar, especialmente debido a que no existen servicios básicos que permitan satisfacer las demandas de los nuevos habitantes que se están proyectando, así como también argumentan una serie de problemáticas fundamentadas en prejuicios acerca de los nuevos grupos sociales que llegarían a vivir a Curauma. Cabe señalar que estos antecedentes nos permiten dar cuenta de que la heterogeneidad socio económica en términos de distribución espacial no necesariamente permite garantizar instancias reales de integración social entre la comunidad, lo cual abre líneas de investigación interesantes de desarrollar sobre la definición de los procesos de inclusión y exclusión social que se han trabajado sobre el concepto de segregación residencial.

En el sector Sur de Curauma es posible encontrar 8 proyectos inmobiliarios, los cuales poseen valores que varían entre 2087 UF a 5700 UF. Estos superan a los que son posibles encontrar en el lado Norte de Curauma debido a que poseen una mayor calidad paisajística por su cercanía al lago, así como mayor acceso a áreas de esparcimiento, recreación y deporte, diferencias que claramente inciden en una diferenciación socio económica entre los sectores. De estos ocho proyectos, seis corresponden a condominios cerrados que garantizan seguridad las 24 horas y poseen una serie de elementos que garantizan la vigilancia de las viviendas.

Así, en el sector Sur se ubicarían los estratos socioeconómicos más altos y en el sector Norte los grupos socioeconómicos de clase media y media baja, develando pautas de distribución espacial y condiciones sociales dentro de la ciudad, típicas de Latinoamérica, donde se suele mostrar una segregación residencial en función de los ingresos de los 


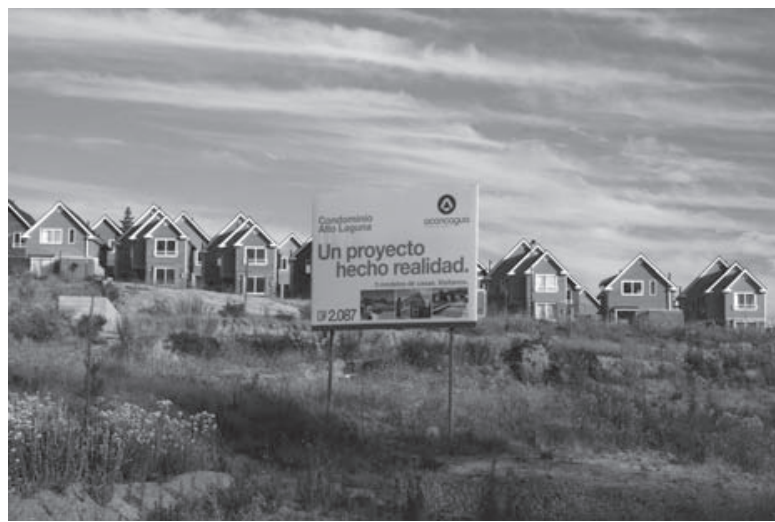

FOTO 1: Condominio Alto Laguna, viviendas desde 2087 UF, cercanas a la orilla de Lago.

Fuente: Elaboración Propia

hogares, creando agrupaciones donde predomina población de ingresos semejantes o superiores ${ }^{17}$.

Finalmente, en la nueva ciudad de Curauma, en el lado Este de la ruta 68, se ha consolidado un sector Industrial en el cual es posible encontrar una serie de industrias enfocadas al desarrollo de diversas actividades como alimentos, tecnología, servicios al puerto de Valparaíso como bodegaje de container. Además de un edificio institucional construido por CORFO, el que viene a consolidar la importancia de Curauma como polo empresarial dentro de la región y que apunta a incentivar la llegada de nuevas empresas al sector.

17 FLóREZ Díaz, Josefina, 2006.

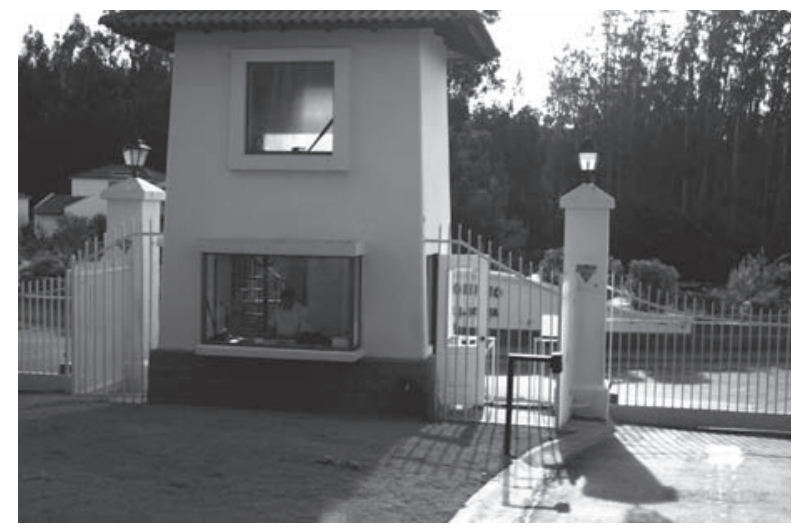

FOTO 2: Control de seguridad en entrada del condominio cerrado "Borde Laguna".

Fuente: Elaboración Propia.

\section{Curauma, ¿una ciudad vallada en el Área Metropolitana de Valparaíso?}

La ciudad de Curauma representa la introducción al país del modelo de suburbio norteamericano, que marca uno de los precedentes de los posteriores proyectos similares que se instauran en el país muy semejantes a las ZODUC.

Entre las diversas tipologías de urbanizaciones cerradas, la más cercana a Curauma es la de una Ciudad Vallada que aún no ha logrado entrar en 


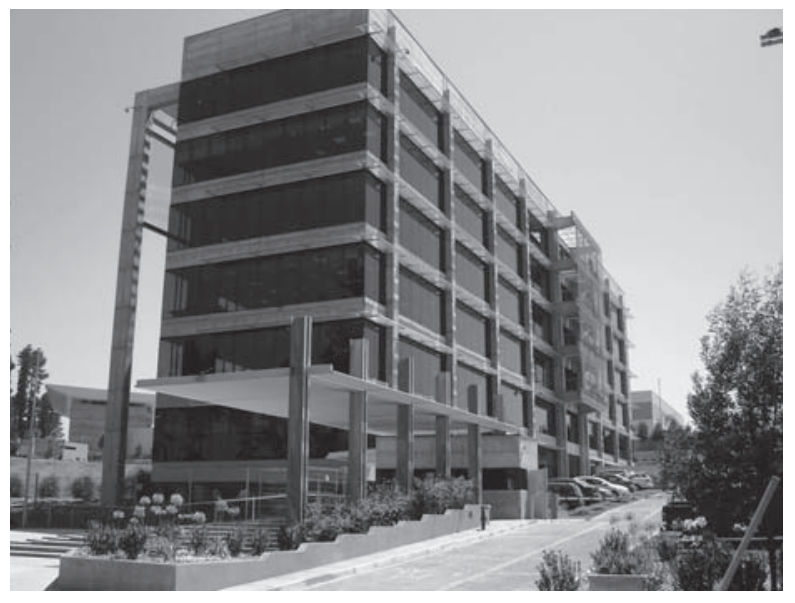

FOTO 3: Edificio CORFO, ubicado en el sector Tecnológico - Industrial de Curauma.

Fuente: Elaboración Propia.

su fase de consolidación, debido a que continúa manteniendo una relación de dependencia funcional con las comunas articuladoras del AMV. Precisamente, Curauma concuerda con el modelo planteado por Hidalgo ${ }^{18}$, si tomamos en cuenta que las "ciudades valladas" hacen referencia a mega proyectos inmobiliarios construidos en distintas fases, cercanos a la periferia de las áreas metropolitanas, constituidas principalmente por viviendas unifamiliares, los cuales se encuentran potenciados por el desarrollo de instrumentos de ordenamiento

18 HIDALGO, Rodrigo. 2004

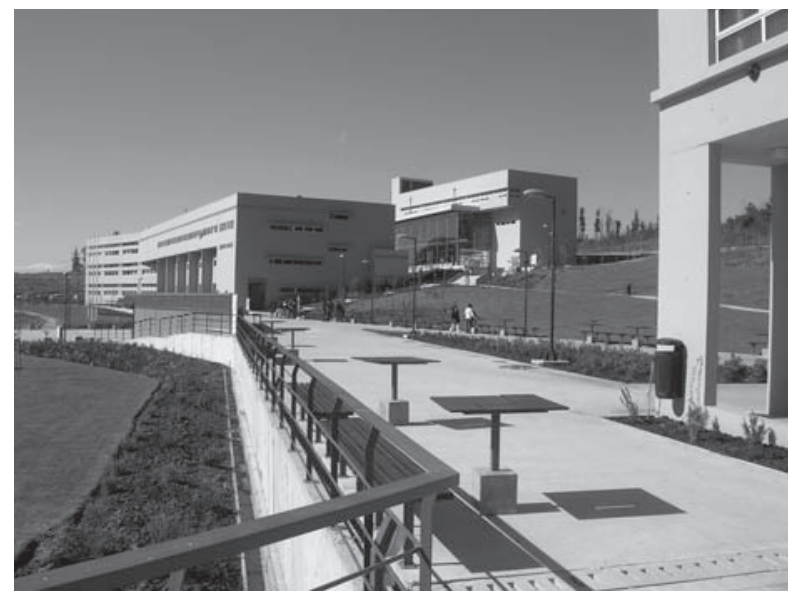

FOTO 4: Edificaciones inauguradas recientemente por la Pontificia Universidad Católica de Valparaíso en Curauma, cercanas a la laguna. Fuente: Elaboración Propia.

territorial especiales y por la excelente conectividad y accesibilidad espacial que le da su cercanía a importantes vías estructurantes. Sin embargo, Curauma no logra cumplir con un elemento fundamental que debe poseer una ciudad vallada, el cual hace referencia a que ésta, "en su diseño debe considerar una amplia gama de equipamientos y servicios, que dan autonomía a estos asentamientos respecto del entorno urbano donde se localizan", lo que en el caso de Curauma aún no ha sido posible de lograr, generando una brecha entre el 
proyecto diseñado y la situación actual presente en este espacio.

A medida que Curauma ha ido creciendo, han comenzado a localizarse en ella una serie de prestadores de servicios privados (Telefonía, Televisión, Seguridad) al ver un mercado cautivo y en potencial crecimiento, resaltando entre ellos aquellos que guardan relación con servicios de seguridad privada, además de la que ya ofrecen los propios condominios. También es posible encontrar supermercados, colegios particulares y un gran complejo universitario de la Pontificia Universidad Católica de Valparaíso denominado Campus Curauma.

Sin embargo, Curauma no ha sido capaz de lograr generar nuevas centralidades urbanas en el contexto del AMV. De esta forma su relación de dependencia con los núcleos urbanos tradicionales se ha mantenido fuertemente, manteniendo una migración pendular diaria para acceder a servicios y a puestos de trabajo. La idea es potenciar el desarrollo demográfico de la ciudad, para lograr llamar la atención de los inversionistas, y la localización de servicios que permitan que Curauma se desarrolle como tal.

Para alcanzar un conocimiento más exhaustivo del número y grado de especialización de las funciones que cumplen los asentamientos pertenecientes al AMV, más las ciudades periféricas de Casablanca y Limache, a objeto de sondear una medida de exclu-

ARTICULO: Procesos de desarrollo urbano-regional y exclusión territorial: Nuevas formas de urbanización en el área metropolitana de Valparaíso. Estudio de caso ciudad de Curauma

Pablo Mansilla 0., Manuel Fuenzalida D. sión territorial en términos de igualdad de oportunidades de acceso a funciones urbanas, se presenta un procedimiento metodológico a partir de los planteamientos de Davies ${ }^{19}$. Estos corresponden a la aplicación de los coeficientes de localización, valores de centralidad e índice funcional. Es importante señalar que la propuesta aquí está limitada a un número reducido de variables, en atributo de su viabilidad de ser levantadas en terreno.

El coeficiente de localización (CL) fue aplicado a siete funciones seleccionadas según criterio de frecuencia existente, las cuales se pueden agrupar en comerciales (bancos y grandes cadenas de supermercados), sociales (consultorios generales y comisaría) y de servicios (colegios, establecimientos de educación superior y farmacias). El propósito es reducir todas las funciones a una base común, para así poder comparar cada tipo de función. La fórmula corresponde a:

$$
C L_{j}=\frac{k * 100}{T}
$$

Donde:

CL ${ }^{j}$ : Coeficiente de localización de la función j.

T: Número de veces que se presenta la función "j" en todo el sistema urbano.

k: Función Constante, unitaria.

Los valores de centralidad (VC) se obtienen a partir de la multiplicación entre el coeficiente de loca-

19 DAVIES, Wayne. 1967. 
lización de cada función y el número de establecimientos de la misma en el núcleo considerado. Así es posible obtener el nivel de focalidad que cumple cada núcleo urbano de acuerdo al tipo de funciones. La fórmula corresponde a:

$$
V C_{i j}=C L_{j} * X_{i j}
$$

Donde:

VC $=$ Valor de centralidad de la función j en el núcleo urbano i.

$\mathrm{CL}_{j}$ : Coeficiente de localización de la función j.

$\mathrm{X}_{i j}$ : Número de establecimientos de la función j presentes en el núcleo urbano i.

Finalmente obtenemos el índice funcional (IF), el cual es el resultado de la sumatoria de todos los valores de centralidad de cada asentamiento urbano. Hallados los valores del índice funcional se pueden conseguir los límites de los escalones jerárquicos (primero, segundo, tercero). La fórmula corresponde a:

$$
I F_{i}=\sum_{j=1}^{m}\left(V C_{j}\right)
$$

Donde:

IFi: Índice funcional del núcleo urbano i.

$\mathrm{VC}^{j}=$ Valor de centralidad de la función j.

Los resultados de la metodología de Davies se presentan en la tabla $n^{\circ} 2$. De ellos se coligen en cuanto al CL que las funciones que ejercen una mayor capacidad de organización territorial son, en orden descendente, comisarías, supermercados y consultorios. Estos sugieren un mayor volumen de desplazamientos por parte de la población residente en el AMV hacia centros urbanos específicos, como por ejemplo, Valparaíso y Viña del Mar, para la obtención de los beneficios que proveen estos tipos de servicios.

Los establecimientos de educación superior, bancos y farmacias, se encuentran en un nivel intermedio de capacidad de organización territorial, lo que implican desplazamientos más acotados para suplir la demanda, debido a que presentan mayor dispersión en el territorio. Finalmente, los establecimientos de educación obligatoria son los que presentan mayor frecuencia en el sistema urbano $(\mathrm{CL}=0,16)$, lo que indica que es un servicio básico instalado en cada uno de los núcleos analizados, independiente de su tamaño poblacional.

Asimismo, podemos mencionar que el nivel de focalidad de los servicios analizados al interior de los asentamientos urbanos no se muestra homogéneo. Sólo en el 50\% de ellos (4) se cubren las siete funciones analizadas, entre las que destacan Viña del Mar y Valparaíso, con valores obtenidos que exceden en forma importante la media de las otras focalidades comunales, demostrando de esta forma, el nivel de hipertrofia del sistema urbano regional.

Bajo esta misma mirada, Curauma y Con-Cón son los núcleos más dependientes y excluidos territorialmente del AMV, destacando el hecho de que ambos carecen de dos servicios, siendo el común entre ellos, el de comisaría, con lo cual los habi- 


\section{ÍNDICES DE LOCALIZACIÓN DE FUNCIONES EN EL AMV}

\begin{tabular}{lrrrrrrrr} 
Asentamiento & \multicolumn{9}{c}{ Valores de Centralidad } & & IF \\
& Bancos & Supermercados & Consultorios & Comisarías & Colegios & E.E.S. & Farmacias & \\
Curauma & 0,00 & 6,90 & 2,78 & 0,00 & 1,28 & 1,96 & 0,97 & 14,85 \\
\hline Con-Cón & 5,19 & 6,90 & 2,78 & 0,00 & 3,35 & 0,00 & 2,97 & 21,19 \\
\hline Casablanca & 3,90 & 3,45 & 2,78 & 11,11 & 3,99 & 0,00 & 0,99 & 26,22 \\
\hline Limache & 3,90 & 6,90 & 2,78 & 11,11 & 5,59 & 1,96 & 2,97 & 35,20 \\
\hline Villa Alemana & 5,19 & 3,45 & 8,33 & 11,11 & 11,18 & 0,00 & 4,95 & 44,22 \\
\hline Quilpué & 9,09 & 20,69 & 8,33 & 11,11 & 17,41 & 7,84 & 8,91 & 83,39 \\
\hline Valparaíso & 28,57 & 24,14 & 38,89 & 33,33 & 25,40 & 43,14 & 30,69 & 224,16 \\
\hline Viña del Mar & 44,16 & 27,59 & 33,33 & 22,22 & 31,79 & 45,10 & 48,51 & 252,70 \\
CL & 1,30 & 3,45 & 2,78 & 11,11 & 0,16 & 1,96 & 0,99 &
\end{tabular}

TABLA 2

Fuente: Elaboración propia.

tantes de dichos núcleos urbanos presentarían un desmedro en la prevención y control de delitos (dada la menor dotación policial), o mayores impedimentos para denunciar la vulneración de sus derechos. Esto sin duda da señales inequívocas de que el desarrollo urbano espontáneo, liderado por el sector inmobiliario, no considera la dotación de ofertas de servicios de interés público.

Si a ello se agrega que Curauma no presenta bancos, queda en evidencia que este núcleo continúa manteniendo una relación de dependencia con las cabeceras del AMV, tanto en temas de acceso a ser-

ARTíCULO: Procesos de desarrollo urbano-regional y exclusión territorial: Nuevas formas de urbanización en el área metropolitana de Valparaíso. Estudio de caso ciudad de Curauma / Pablo Mansilla Q., Manuel Fuenzalida D. vicios financieros por lo que el CBD se mantiene en Valparaíso, como en temas de seguridad pública, demandando aquellos que se encuentran disponibles en Placilla, saturando aún más la escasa cobertura de carabineros disponible.

Sumado a lo anterior, al realizar una representación esquemática de los asentamientos urbanos por jerarquía funcional ${ }^{20}$, se manifiestan dos elementos relevantes desde el punto de vista de sus funciones. El primero de ellos, es que indudablemente se da cuenta del proceso histórico de desarrollo urbano que ha marcado al sistema de

20 Ver foto 3 y foto 4. 
asentamientos del AMV, especialmente en el desarrollo espontáneo en la periferia, de conjuntos de viviendas tanto formales como informales, sin la adecuada provisión de funciones urbanas básicas. Así, la exclusión adopta una forma fundamentalmente espacial, en donde las oportunidades de acceso a funciones urbanas se encuentran determinadas por la posición geográfica, tanto de los puntos oferentes como de los puntos consumidores. El segundo de ellos, es que se reafirma la praxis de que un mayor volumen de población en un centro urbano determina la instalación de un alto número de servicios que buscan aprovechar las ventajas de localización para captar mayores cuotas de mercado (interés privado), o ser eficientes (interés público) en la satisfacción de necesidades básicas.

\section{Nuevas formas de urbanización y exclusión territorial}

A pesar de que existen diversos enfoques con los cuales puede ser abordada la exclusión social, Cebollada ${ }^{21}$ menciona que de forma general este término puede ser entendido a partir de la propuesta conceptual realizada desde las ciencias sociales por Tourain ${ }^{22}$ en el contexto social de las desigual-

21 CEBOLLADA, Ángel, 2006.

22 TOURAINE, 1991 (Citado en CEBOLLADA, Ángel, 2006). dades presentes en las sociedades industriales y el retroceso de los estados de bienestar, para referirse a "alguna cosa o alguien que queda fuera de algo, en contraposición a los que están dentro". En este mismo sentido, podemos decir que la "exclusión territorial" puede ser entendida como una de las formas en las que se manifiestan los procesos de exclusión social en su dimensión material, cuando un individuo o un grupo encuentra diferencias significativas en sus posibilidades de acceso a los beneficios de la ciudad debido a las restricciones impuestas por las condiciones estructurales o de emplazamiento que poseen.

El desarrollo del proyecto inmobiliario generado por Curauma representa una nueva forma de urbanización que genera una ruptura de las características primigenias que es posible encontrar en el contexto del AMV, potenciando la polarización de los estratos socio económicos medios y altos en Curauma y generando fuertes contrastes sociales con sectores aledaños como Placilla y las periferias pobres de las ciudades que componen el AMV. Sin embargo, tal como hemos visto, las relaciones de dependencia con los centros urbanos estructurantes en el AMV de Viña del Mar y Valparaíso se han mantenido, lo cual nos permite presumir que el desarrollo y la vigencia de ambos centros urbanos como articuladores no se encuentra amenazada. 


\section{DIAGRAMA DE ASENTAMIENTOS URBANOS POR JERAROUÍA FUNCIONAL}

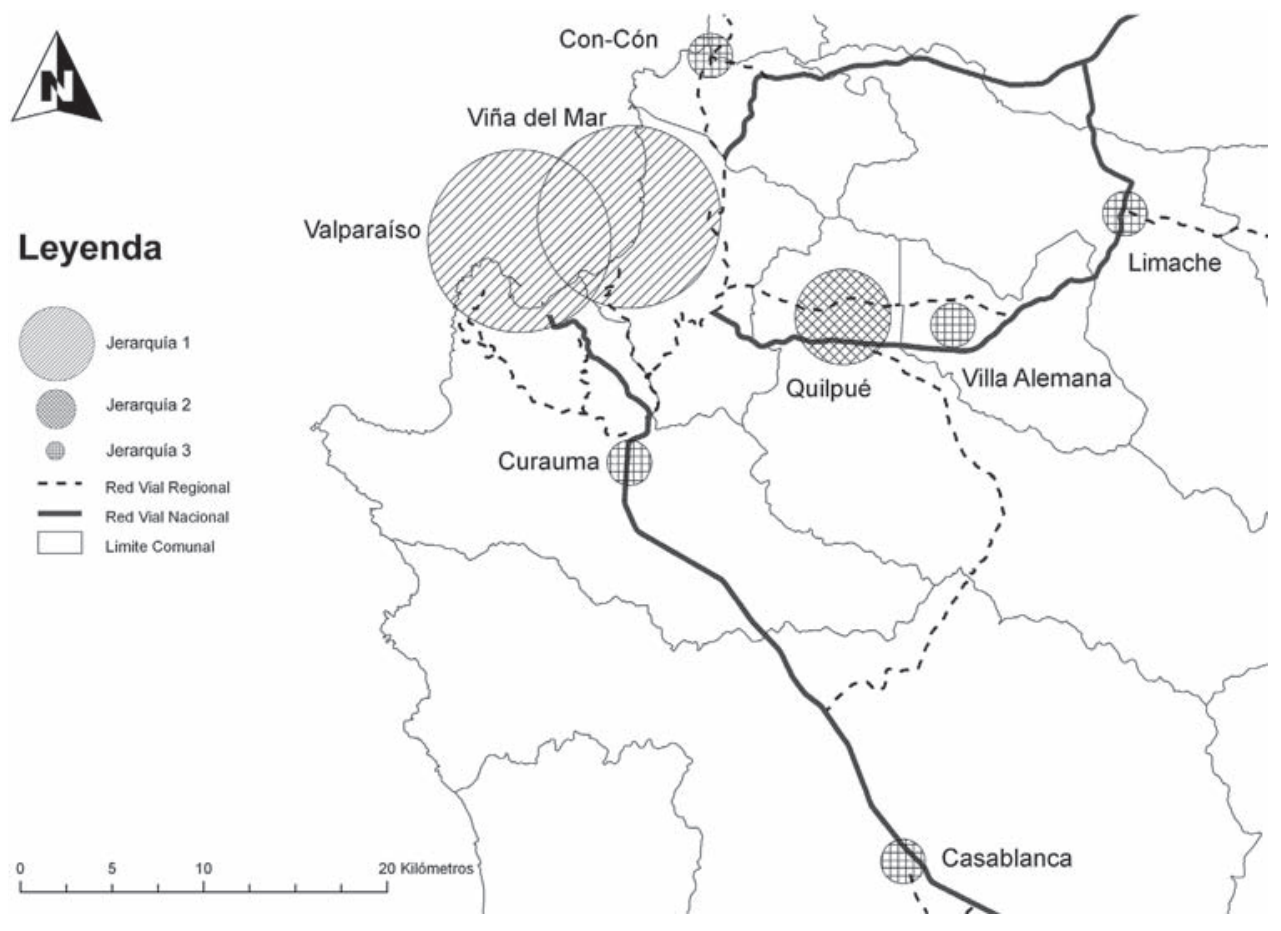

FIGURA 2

Fuente: Elaboración propia.

De esta forma, Curauma tributa de los servicios que prestan las otras ciudades y asentamientos cercanos, sin que esta dinámica se transforme en una relación recíproca que potencie la complementariedad de funciones entre los centros urbanos, pues al constituirse como una Ciudad Vallada, no facilita el acceso (tanto a las barreras materiales que impone, como también culturales) a otros tipos de servicios que ofrece, como ambientales o recreativos. Esta relación unidireccional puede entenderse como una forma de "exclusión territorial" que evidencia el beneficio de tan solo un grupo 
favorecido de individuos. Esta dicotomía se hace especialmente evidente cuando se constata que Curauma, a pesar de los fuertes contrastes sociales con sectores aledaños como Placilla, por su cercanía geográfica, tributa de los ya escasos servicios disponibles en el sector de Placilla. Si a ello se suman los proyectos de vivienda social desarrollados en Curauma y los futuros proyectos que se pretenden impulsar, las posibilidades de acceso a servicios de interés público de calidad se dificulta aún más, especialmente para la población en situación de vulnerabilidad económica ${ }^{23}$, pues son ellos los que en definitiva tienen que invertir más recursos en tiempo, dinero o esfuerzo para poder desplazarse hacia los centros oferentes que satisfagan sus necesidades. Estas dinámicas contribuyen a generar procesos de exclusión territorial, en el que los grupos más vulnerables no logran tener igual acceso de uso y disfrute que los otros grupos sociales, a servicios y espacios de calidad en la ciudad, principalmente, debido a que ellos muestran mayores dificultades para especializar su demanda, referida a la capacidad de hacer uso efectivo de alguna actividad o servicio ${ }^{24}$.

Esto sin duda da señales inequívocas de que el desarrollo urbano espontáneo, liderado por el sector inmobiliario, es uno de los principales facto-

23 Clase media y media baja.

24 FUENZALIDA Díaz, Manuel; MORENO Jiménez, Antonio, 2009.

120 revista invi № 69 / Agosto 2010 / Volumen N ํ 25: 103-123 res que inciden en la exclusión territorial, lo cual encuentra una explicación válida en el repliegue del Estado y de sus instituciones en los procesos de planificación y gestión del desarrollo urbano. Al flexibilizar el rol que cumple el estado y traspasar sus competencias a las lógicas de mercado, se garantiza la posibilidad de que existan instancias de acceso para todas las personas para la satisfacción de sus necesidades a diferentes costos, sin embargo, estas posibilidades de acceso no son iguales para todos e implican esfuerzos dispares entre los habitantes de la ciudad, así como también implican calidades de acceso diferenciadas y por lo tanto excluyentes. En este sentido, Janoschka ${ }^{25}$ se refiere a esta idea argumentando que "el abandono de la gestión y control del desarrollo urbano por parte del Estado y su apropiación por parte de actores privados ha generado como resultado la aparición de formas urbanas comercializables, redituables y valiosas para el mercado" 26 , las cuales están básicamente dirigidas a los ganadores de las transformaciones económicas. A su vez, aquella parte la población que no ha podido obtener las herramientas necesarias para poder integrarse dentro de los nuevos modelos de desarrollo se han transformado en los actores perdedores en el juego del mercado, éstos se encuentran en un situación

25 JANOSCHKA, Michael, 2002.

26 Es decir, Shopping Malls, Urban Entertainment Center, escuelas privadas y complejos residenciales cerrados, vigilados y de acceso vedado al público en general". 
que los limita en cuanto a la capacidad de elección y acceso a ciertos espacios de la ciudad definidos para los estratos socioeconómicos altos.

Sin embargo, es posible identificar que a pesar del evidente repliegue del Estado en la planificación del desarrollo urbano, éste aún sigue tratando de incidir en la organización del desarrollo territorial por medio de incentivos asociados a grandes inversiones en infraestructura que llamen la atención o consoliden iniciativas llevadas a cabo por el sector privado. Este cambio de estrategia en la producción del desarrollo urbano adoptada por el Estado se enmarca dentro de un "modelo de desarrollo burocrático", el cual, tal como lo menciona Guerra $^{27}$, busca "asentar el control socio político a través de la generación de grandes proyectos comunes, principalmente relacionados al desarrollo de equipamiento e infraestructura", justificando el accionar del Estado, dando muestra del progreso (estimado de forma material) que ha logrado impulsar por medio de grandes obras. Esta racionalidad en la que opera la lógica del Estado en cuanto al desarrollo territorial es explicada también por De Mattos $^{28}$, aludiendo que en las últimas décadas el Estado se ha encargado de impulsar una serie de obras de infraestructura en el interior y exterior de las ciudades, estos elementos generan la valoración de la tierra urbana, como es el caso de las carre-

27 GUERRA Rodríguez, Carlos, 1997.

28 De Mattos, 1999 (Citado por HIDALGO, Rodrigo. et. al. 2007). teras autopistas concesionadas, las que conectan a los llamados artefactos de la globalización. Asimismo, Borsdorf e Hidalgo ${ }^{29}$ reafirman esta idea mencionando que "el interés estatal por incentivar estos aparatos, se debe al hecho de que estos elementos contienen una fuerte carga simbólica, pues representan la modernización del país y su integración al mundo desarrollado". Es decir, el Estado mediante estas iniciativas no busca transformarse en el eje conductor del desarrollo territorial, sino que más bien intenta mediante estos incentivos potenciar el desarrollo de proyectos por parte del mundo privado para dar cuenta del progreso en términos materiales. En el caso de Curauma, los incentivos que ha generado el Estado a través de CORFO y fondos de Investigación para dar un incentivo a la generación de un polo de desarrollo industrial y tecnológico en la zona son un claro ejemplo de cómo operan estas racionalidades. Asimismo la elaboración de un plan seccional especial por parte del Municipio y la habilitación de infraestructura vial para su conexión con la ruta 68 representan un aporte a la consolidación del proyecto.

Los antecedentes hasta aquí recopilados, dan cuenta de ciertos rasgos comunes en los procesos de urbanización que han desarrollado durante las últimas décadas en la región de Valparaíso y en Chile

29 BORSDORF, Axel; HIDALGO, Rodrigo, 2009. 
como resultado de las lógicas de la producción del espacio urbano desde la adopción del enfoque neo liberal. Curauma se presenta como un caso de estudio interesante de seguir profundizando en otras investigaciones que nos permitan explorar la manera en que estas nuevas formas de urbanización entran en relación con otros asentamientos del AMV y los procesos de exclusión social asociados a estas dinámicas tanto en la dimensión material, como cultural. De esta forma, a partir de los resultados presentados, se pretende abrir caminos para nuevas investigaciones que nos permitan profundizar en la discusión en torno a los impactos generados por las nuevas formas de urbanización en la Región de Valparaíso, el rol que deben cumplir los actores que participan en el proceso del desarrollo urbano y la forma en la cual podemos repensar el desarrollo territorial en la búsqueda de procesos de desarrollo urbano y territorial realmente incluyentes.

\section{Bibliografía}

BORSDORF, AXEL; HIDALGO, RODRIGO. El Crecimiento Urbano en Europa: Conceptos, Tendencias y Marco Comparativo Para el Área Metropolitana de Santiago de Chile. Revista Estudios Geográficos. Enero-Junio del 2009. Vol. LXX, No 266, págs. 181-203. ISSN 0014-1469.

122 revista invi № 69 / Agosto 2010 / Volumen № 25: 103-123
BORSDORF, AXEL. Cómo modelar el desarrollo y la dinámica de la ciudad latinoamericana. Revista Eure. Mayo 2003. Vol. 29, No 26, págs. 37-49. ISSN 0250-7161.

CEBOLLADA, ÁNGEL. Aproximación a los procesos de exclusión social a partir de la relación entre el territorio y la movilidad cotidiana. Doc. Anales de Geografía. Año 2006, N 48, págs. 105-121.

DAVIES, WAYNE. Centrality and the central place hierarchy. Urban Studies. Enero 1967. Vol. 4 (1). págs. 61-79.

DÍAZ ORUETA, FERNANDO, et al. Ciudad, territorio y exclusión social. Las políticas de recualificación urbana en la ciudad de Buenos Aires. Revista española de investigaciones sociológicas Reis. Año 2003, No 103. págs. 159-185. ISSN 0210-5233.

FLÓREZ DÍAZ, JOSEFINA. El proceso de toma de decisión residencial: Un modelo conceptual y los atributos que son valorados. Revista Argos. Junio 2006, Vol. 23 No 44, págs. 32-53. ISSN 0254-1637.

FUENZALIDA DÍAZ, MANUEL; MORENO JIMÉNEZ, ANTONIO. Propuesta metodológica para establecer el patrón territorial de status socio-económico de la población, basada en pequeñas unidades espaciales estándar. Aplicación a la Región de Valparaíso (Chile). En Conferencia Iberoamericana en Sistemas de Información Geográfica. $12^{\circ}$, 2009, San José, Costa Rica: Universidad Nacional de Costa Rica. 
GUERRA RODRÍGUEZ, CARLOS. Nueva estrategia neoliberal: La participación ciudadana en Chile. 1 ed. Cuernavaca, México, Centro Regional de Investigaciones Multidisciplinarias-UNAM. 1997. 511 p. ISBN 109683646352.

HIDALGO, RODRIGO. De los pequeños condominios a la ciudad Vallada: Las Urbanizaciones Cerradas y la Nueva Geografía Social en Santiago de Chile (1990-2000). Revisa Eure. Diciembre 2004, Vol. 30, No 91, págs. 29-52. ISSN 0250-7161.

HIDALG0, RODRIGO; BORSDORF, AXEL. Puerto Abierto ¿Ciudad Cerrada? Transformaciones SocioEspaciales en la estructura urbana del área metropolitana de Valparaíso. Revista Geográfica de Valparaíso. Año 2005. Vol. No 36, págs. 189-206. ISSN 0716 - 1905.

HIDALGO, RODRIGO; GONZÁLES, CAROLINA. LOS condominios y las nuevas formas de construcción del espacio urbano: Efectos en la Geografía Social y Percepción de los residentes. El caso de las comunas de Quilpue y Villa Alemana, V Región de Valparaíso. Revista Geográfica de Valparaíso. Año 2004, Vol. No 35, págs. 129-140. ISSN 0716- 1905.

HIDALGO, RODRIGO. et. al. El desborde de la Vivienda Social en Valparaíso: Efectos Socioeconómicos y
Espaciales (1992 - 2005). Revista Geográfica de Valparaíso. Semestre I año 2007, Vol. N³9, págs. 71-84. ISSN 0716 - 1905.

HIDALGO, RODRIGO; BORSDORF, AXEL; SÁNCHEZ, RAFAEL. Globalización, neoliberalismo y producción inmobiliaria: hacia la ciudad vallada en las capitales del cono sur. El caso de Santiago de Chile. En: Encuentro de Geógrafos de América Latina $\left(10^{\circ}, 2005\right.$, Sao Paulo, Brasil). Universidad de São Paulo.

JANOSCHKA, MICHAEL. El nuevo modelo de la ciudad latinoamericana: fragmentación y privatización. Revista EURE. Diciembre 2002. Vol. 28, No 85. págs. 11-20. ISSN 0250-7161.

PARNREITER, CHRISTOF. Tendencias de Desarrollo en las Metrópolis Latinoamericanas en la era de la Globalización: Los casos de Ciudad de México y Santiago de Chile. Revista Eure. Mayo 2005, Vol. 31, No 92, págs. 5-28. ISSN 0250-7161.

WARNER, KEE Y NEGRETE, JORGE. Las maquinarias de Urbanización en un país en vías de desarrollo: El caso del Gran Valparaíso en Chile. Revista Geográfica de Valparaíso, Año 2001-2002, Vol. No 32 - 33, págs. 56-89. ISSN 0716 - 1905. 